Esta revista forma parte del acervo de la Biblioteca Jurídica Virtual del Instituto de Investigaciones Jurídicas de la UNAM

\title{
ESTRATEGIAS PARA ATENDER EL EMPLEO JUVENIL EN LA ECONOMÍA INFORMAL*
}

\author{
STRATEGIES FOR YOUTH EMPLOYMENT \\ IN AN INFORMAL ECONOMY
}
STRATEGIES POUR ASSISTER L'EMPLOI DE LA JEUNESSE DANS L'ECONOMIE INFORMELLE

\section{María Ascensión MORALES RAMÍREZ**}

RESUMEN: La población juvenil enfrenta obstáculos en su inserción laboral y social. A nivel internacional se reconoce que el déficit de trabajo decente en este sector es de gran magnitud y amplia heterogeneidad. Dentro de los grupos críticos, se ubican aquellos que trabajan en la economía informal, fenómeno que se incrementa cada vez más. Ante esta situación, se pugna por políticas diferenciadas para cada grupo juvenil, en donde si bien el crecimiento económico es esencial para generar empleos de calidad también se requieren políticas sociolaborales a fin de atender la problemática juvenil. Entre las estrategias para reducir o eliminar en esta población el empleo en la economía informal destacan: programas de incentivos a la formalización, piso básico de protección social, educación, formación y competencias e iniciativa empresarial.

Palabras clave: Trabajadores jóvenes, empleo juvenil, empleo informal, transición al trabajo formal.

ABSTRACT: Youth come up against many obstacles in their search for employment and social integration. The lack of decent work for this sector of the population is recognized internationally as a prevalent and manifold problem. Among the most critical are the groups working in

* Recibido el 1o. de septiembre de 2015 y aceptado para su publicación el 1o. de abril de 2016.

** Profesora de Derecho del trabajo y la seguridad social en la Facultad de Derecho de la UNAM. 
the informal economy, a phenomenon that ever more common. In view of this situation, distinct policies must be passed for specific groups of young people. While economic growth is essential to the creation of quality employment, social-labour policies are also needed to address youthrelated issues. Many strategies aimed at reducing or eliminating informal employment are in place for this sector of society, including incentive programs for transitioning to the formal economy, a basic social protection floor, education, training and competencies, and entrepreneurship.

Key Words: Young workers, youth employment, informal work, transition to formal employment, working conditions for youth.

RÉSUMÉ: La population jeune affronte des obstacles dans l'insertion professionnelle et sociale. Au niveau international on reconnaisse le déficit d'emplois décents pour la jeunesse, un secteur de grand impact et largement hétérogène. Dans les groupes clefs, il y a quelques secteurs qui travaillent dans l'économie informelle, un phénomène de croissance rapide. Face à cette situation, on fait un appel pour l'implémentation des politiques différenciées pour chaque groupe, tandis que la croissance économique est essentiel pour la création d'emplois de qualité, d'autre part, il faut appliquer des politiques publiques socio-professionnelles à fin d'assister les problèmes de la jeunesse. Parmi les stratégies pour diminuer ou supprimer le travail informel dans ce secteur, les tactiques qui visent remarquablement sont: des programmes pour encourager la formalisation du travail, un seuil de protection social, d'éducation, de formation professionnelle et de concurrence, et des compétences et initiatives d'entrepreneuriat.

Mots-clés: Travailleurs jeunes, emploi de jeunesse, emploi informel, formalisation des emplois.

SUMARIO: I. Introducción. II. Aspectos conceptuales. III. Situación laboral juvenil. IV. Estrategias internacionales. V. Intervenciones nacionales. VI. Conclusiones. VII. Bibliografía.

\section{INTRODUCCIÓN}

as tendencias demográficas, los cambios en el mundo del trabajo y las crisis económicas han tenido expresiones particulares y complejas para la población juvenil. De acuerdo con la Organización Internacional del 
Esta revista forma parte del acervo de la Biblioteca Jurídica Virtual del Instituto de Investigaciones Jurídicas de la UNAM

Trabajo (OIT) en 2013, de los 108 millones de personas entre 15 y 24 años de edad en la región, el 52\% enfrenta algún problema de inserción laboral y social (56 millones). Si bien los diversos colectivos juveniles encaran dificultades y necesidades diferentes dados los altos niveles de heterogeneidad de este sector, tres situaciones revisten particular interés: los desempleados (7.8 millones), los que no estudian ni trabajan (21.8 millones) y los que se insertan al mundo laboral en forma precaria y/o vulnerable (48.3 millones).

Cada uno de estos grupos amerita y es objeto de análisis específicos, pues el desempleo juvenil es tres veces superior a la tasa de los adultos, el fenómeno de los que no estudian ni trabajan pone de manifiesto los altos costos sociales de la marginación de los jóvenes de dos de los principales mecanismos de integración social: la escuela y el trabajo; y los que trabajan, en su gran mayoría, se ubican en la economía informal, lo que implica bajos ingresos, inestabilidad y desprotección social.

Desde 2002 el fenómeno denominado en principio "informalidad" ha adquirido considerable importancia en los debates internacionales. ${ }^{1}$ Según la OIT, la economía informal (expresión más amplia) es, ante todo, una cuestión de gobernanza. El crecimiento de este fenómeno se debe: a) a políticas macroeconómicas y sociales inadecuadas, ineficaces, encubiertas o mal aplicadas, y b) la falta de marcos jurídicos e institucionales favorables y de buena gobernanza para la aplicación correcta y eficaz de las políticas y leyes.

La mayoría de las personas que se incorporan a la economía informal no lo hacen por elección, sino como consecuencia de la falta de oportunidades en la economía formal y por no contar con medios de subsistencia. Superar el empleo en la informalidad es el principal desafío en materia de desarrollo en todos los países y es obligado para la consecución del trabajo decente de los jóvenes.

Dado que a nivel regional y nacional el tema del empleo en la "economía informal" es de tal magnitud, éste ha cobrado mayor importancia en el debate laboral y, hoy en día, es objeto de una nueva norma internacional del trabajo la Recomendación 204, sobre la Transición de la economía informal a la economía formal,

1 La economía informal fue objeto de particular atención en la undécima Reunión Regional Africana de la OIT en 2007. Asimismo, fue el tema principal del taller sobre la economía informal organizado conjuntamente por la Unión Africana y la OIT en 2008. Igualmente, está siendo objeto de debate en las discusiones sobre la agenda para el desarrollo con posterioridad a 2015. 
adoptada en la 104a. reunión de la Conferencia Internacional del Trabajo el 12 de junio de 2015, ya que se reconoce que la incidencia de este fenómeno representa un importante obstáculo para el desarrollo incluyente y el cumplimiento del Estado de derecho; asimismo, porque tiene consecuencias negativas para el desarrollo, la sostenibilidad de las empresas, la protección social de los trabajadores y sus condiciones de trabajo, los ingresos públicos, la solidez de las instituciones y la competencia leal en los mercados nacionales e internacionales. ${ }^{2}$

En razón de lo anterior, el objetivo del presente estudio se centra en revisar la heterogeneidad del empleo en la economía informal, así como el enfoque integrado de las políticas para reducir o eliminar este fenómeno en el espacio juvenil. En primer lugar, en forma sumaria se abordan aspectos conceptuales necesarios para el tema. En segundo lugar, se presenta la situación laboral juvenil regional y nacional en donde se da cuenta de la vulnerabilidad laboral de este sector de la población. En tercero, se analizan las estrategias internacionales formuladas para atender la problemática y, por último, se exponen las iniciativas nacionales recientes para enfrentar el fenómeno.

\section{ASPECTOS CONCEPTUALES}

A lo largo de este estudio se usarán una serie de conceptos relacionados con el empleo, que en todos los casos se trata de temas multifacéticos y complejos sobre los cuales resulta conveniente realizar algunas aproximaciones a sus significados, conscientes de que no son figuras jurídicas.

\section{Precariedad del empleo}

No existe una definición consensuada en virtud de lo ambiguo y multifacético del término, además de que se manifiesta de diversas formas, según el país, región y mercados laborales. No obstante, se reconocen algunas características:

2 OIT, La transición de la economía informal a la economía formal, Informe V (2), Conferencia Internacional del Trabajo, 103a. reunión, Ginebra, 2014, p. 87; Recomendación sobre la transición de la economía informal a la economía formal, núm. 204, 2015. 
Esta revista forma parte del acervo de la Biblioteca Jurídica Virtual del Instituto de Investigaciones Jurídicas de la UNAM

- El trabajo se realiza en un horizonte a corto plazo o con un elevado riesgo de perderse: baja calidad, inestabilidad, incertidumbre y desprotección laboral, y de seguridad social por parte de los trabajadores.

- Se expande a medida que los empleadores descubren nuevas formas de evadir regulaciones o encuentran resquicios legales en ellas que les permiten incrementar la rentabilidad de su empresa.

- Se expresa en inestabilidad, la que se traduce en la tendencia a sustituir a los trabajadores de planta con contratos indefinidos por trabajadores temporales, a plazo fijo o bien subcontratados; en disminuir la parte fija del salario y en aumentar la porción variable vinculada a la producción; en niveles salariales en el mínimo o bajo el mínimo; en la no dependencia de un único e identificable empleador.

- Se aprecia en labores que no se realizan necesariamente en las instalaciones de la empresa; con poca protección a la integridad física y psicológica, con escasa o nula posibilidad de ejercer derechos sindicales y de negociar colectivamente. ${ }^{3}$

\section{Vulnerabilidad del empleo}

Se entiende como la probabilidad incrementada de perder la inserción social alcanzada, en el grado que se tuviera, o de no conocerla para los que nunca la han tenido. ${ }^{4}$ Los trabajadores se encuentran más a merced de los ciclos de la economía y no pueden hacer frente a las vicisitudes que los afectan a ellos y sus familias.

\section{Empleo vulnerable}

Es un indicador utilizado por la OIT, 5 para medir la proporción de trabajadores por cuenta propia y los que laboran en industrias familiares sobre los empleados totales.

3 Revista Electrónica Temas Laborales, núm. 5, disponible en: bttp:/ / wmw.dt.gob.cl/ documenta cion/1612/articles0335-Tema laboral-Precarizacdelemokeo. Un mal moderno.pdf.

4 Abdala, Ernesto, "Innovaciones en la formación profesional desde la óptica de sus repercusiones y efectos en la inserción profesional de los jóvenes", Formación profesional, Madrid, Organización de Estados Iberoamericanos, 2013, p. 116.

5 OIT, Indicadores clave del mercado de trabajo, 5a. ed., Ginebra, 2007. 
El organismo internacional considera que estas categorías de trabajadores son particularmente vulnerables cuando se trata tanto de riesgo económico y de fortaleza de los mecanismos institucionales. Estos trabajadores son más propensos a: 1) falta de acuerdos contractuales que pueden conducir a la inseguridad laboral, y 2) son improbables de beneficiarse de la seguridad social, la salud o la cobertura por desempleo. ${ }^{6}$

\section{Informalidad del empleo}

Existen dificultades conceptuales y normativas derivadas de la gran diversidad de situaciones, perfiles y conceptos acerca del fenómeno de la informalidad y que dan lugar a diferentes expresiones, a saber: economía informal, sector informal, empleo informal y el propio término "informalidad". 7 Estos conceptos se refieren a diferentes aspectos de la informalidad en el empleo y a diferentes objetivos.

\section{Economia informal}

A partir de las conclusiones sobre el trabajo decente y la economía informal adoptadas por la Conferencia Internacional del Trabajo (CIT) en 2002, se acuñó el concepto de "economía informal", 8 por ser más amplio que el término "sector informal" que se había empleado desde hace cuatro décadas en el debate sobre las políticas de desarrollo. Se consideró que el concepto de economía informal comprende actividades de diversos sectores, situaciones, causas y plantea diversos problemas que requieren soluciones diferentes.

No obstante, no existe una definición consensuada sobre esta expresión pero, por lo general, se entiende que abarca una gran variedad de trabajadores, empresas y de empresarios con características identificables. Al respecto,

6 OIT, Empleo vulnerable, p. 83.

7 Cfr. Sánchez-Castañeda, Alfredo, Economía informaly empleo: una aproximación desde el derecho del trabajo, documentos de trabajo, México, Instituto de Investigaciones Jurídicas, 2013.

8 En 2001, el director general de la OIT hizo referencia al tema de la economía informal en la Memoria presentada a la Conferencia sobre la reducción del déficit de trabajo decente, en donde señalaba la falta de protección, organización y de voz en el trabajo de quienes laboraban en la economía informal. 
Esta revista forma parte del acervo de la Biblioteca Jurídica Virtual del Instituto de Investigaciones Jurídicas de la UNAM

en las conclusiones de la CIT de 2002, se señaló que el término “economía informal" hace referencia: ${ }^{9}$

\begin{abstract}
$\mathrm{Al}$ conjunto de actividades económicas desarrolladas por los trabajadores ${ }^{10}$ y las unidades económicas ${ }^{11}$ que, tanto en la legislación como en la práctica, están insuficientemente contempladas por sistemas formales o no lo están en absoluto, es decir, las actividades de las personas o empresas no están recogidas por la ley, lo que significa que se desempeñan al margen de ella; o no están contempladas en la práctica, o sea, que si bien las personas operan dentro del ámbito de la ley, ésta no se aplica o no se cumple; o la propia ley no fomenta su cumplimiento por ser inadecuada, engorrosa o imponer costos excesivos. $^{12}$
\end{abstract}

En ningún caso, el concepto abarca actividades ilícitas, tal como se definen en los tratados internacionales pertinentes. ${ }^{13}$ El trabajo en la economía informal puede realizarse en el sector formal, en las empresas del sector informal o en los hogares. ${ }^{14}$

\title{
6. Informalidad
}

Tampoco existe un consenso sobre la definición del término "informalidad", pero se entiende por éste la falta de registro de las empresas, de cobertura de seguridad social o de contratos de trabajo. El consenso alcanzado entre la OIT y expertos internacionales reconocen que el concepto tiene dos dimensiones, las cuales se encuentran integradas y son complementarias: ${ }^{15}$

9 OIT, Conclusiones sobre el trabajo decente y la economía informal, Conferencia Internacional del Trabajo, 90a. reunión, Ginebra, 2002.

10 Por cuenta propia que ejercen su actividad en sus propias empresas del sector informal y asalariados con empleos informales que trabajan en empresas del sector formal.

11 Unidades de producción que emplean mano de obra contratada así como aquellas que pertenecen a personas que trabajan por cuenta propia, ya sea en solitario o con la ayuda de miembros de la familia no remunerados y que son explotadas por ellas.

12 OIT, Resolución sobre el trabajo decente y la economía informal, Ginebra, 2002, párr. 3.

13 Producción y el tráfico de drogas ilegales contempladas en el derecho penal y que por lo cual, no pueden estar reglamentadas en la legislación laboral o comercial.

14 No obstante, el término "economía informal" suele restar importancia a los vínculos, las zonas grises y la interdependencia existente entre las actividades formales y las informales.

15 OIT, El empleo informal en México: situación actual, políticas y desafíos, 2014, p. 4. 
1) Dimensión referida al tipo o naturaleza de la unidad económica. Se dedica a la producción de bienes y/o servicios para el mercado y opera a partir de los recursos de un hogar, sin llevar los registros contables básicos. Se trata de un sector informal o de negocios en pequeña escala no registrada y de empleo vinculado a dicho sector. Este enfoque, aplica a las modalidades de trabajo independiente (empleadores y trabajadores por cuenta propia).

2) Dimensión desde la perspectiva laboral. Se refiere a todo trabajo que se realice sin contar con el amparo del marco legal o institucional, sin importar si la unidad económica que utiliza sus servicios son empresas o negocios no registrados de los hogares o empresas formales. Este enfoque aplica a la modalidad de trabajo dependiente.

Dentro de los diversos aspectos referidos a la "informalidad", se ubican el empleo en el sector informal y el empleo informal. Éstos no son sinónimos, sino complementarios útiles para describir y analizar el fenómeno.

\section{Sector informal}

La OIT introdujo en el debate sobre las políticas de desarrollo el concepto de "sector informal, en un informe publicado en 1972.16 En 1991, la Conferencia Internacional del Trabajo había puesto de relieve la necesidad de abordar el "dilema del sector no estructurado". ${ }^{17}$

El término sector informal se refiere a las empresas informales y, por ello, al empleo generado en un grupo de unidades de producción, que forman parte del sector de los hogares como empresas, es decir, dedicadas a la producción de bienes o a la prestación de servicios que no constituyen una unidad jurídica independiente del hogar propietario ni de los miembros del mismo y que no llevan una contabilidad completa. ${ }^{18}$

16 OIT, Employment, Incomes and Equity: A Strategy for Increasing Productive Employment in Kenya, Ginebra, 1972.

17 OIT, El dilema del sector no estructurado, memoria del director general, parte I, Conferencia Internacional del Trabajo, 78a. reunión, Ginebra, 1991.

18 OIT, Trabajo decente y juventud en América Latina. Políticas para la acción, Lima, 2013, pp. 162 y 163 . 
Esta revista forma parte del acervo de la Biblioteca Jurídica Virtual del Instituto de Investigaciones Jurídicas de la UNAM

\section{Empleo informal}

El concepto se refiere a los empleos que incluyen a asalariados, ya sea que estén empleados por empresas del sector formal, ${ }^{19}$ por empresas del sector informal o por hogares en el carácter de trabajadores domésticos asalariados.

En la decimoséptima Conferencia Internacional de Estadísticos del Trabajo (CIET) celebrada en 2003, se adoptaron las directrices sobre una definición estadística del empleo informal: ${ }^{20}$

Comprende en número total de empleos informales, ya sea que se ocupen en empresas del sector formal, empresas del sector informal, o en hogares, durante un período determinado. Esto abarca: trabajadores por cuenta propia y empleadores de dueños de sus propias empresas del sector informal; trabajadores familiares auxiliares, independientemente de si trabajan en empresas del sector formal o informal; asalariados que tienen empleos informales, ya sea que estén empleados por empresas del sector formal, por empresas del sector informal o por hogares que les emplean como trabajadores domésticos asalariados, miembros de cooperativas de productos informales, $y$ trabajadores por cuenta propia que producen bienes exclusivamente para el propio uso final de su hogar. ${ }^{21}$

Con base en el análisis de los aspectos conceptuales y para los efectos del presente estudio se empleará el término "economía informal”, por ser más amplio y en nuestra opinión constituye el género del fenómeno. A partir de ello, se puede señalar que las diferencias entre los trabajadores de la economía informal se observan en los siguientes rubros:

a) Ingresos: nivel, regularidad, estacionalidad.

b) Situación en el empleo: asalariados, empleadores, trabajadores por cuenta propia, ocasionales, domésticos.

19 En donde los trabajadores subordinados laboran bajo modalidades en las que se alude el registro ante la seguridad social, situación que cada vez más está ganando terreno.

20 OIT, La transición de la economía informal a la economía formal, Informe V (1), Conferencia Internacional del Trabajo, 103a. reunión, 2014, p. 6.

21 Dada la gran diversidad de las situaciones de empleo informal, observadas en diferentes países, en las directrices de la CIET se prevén pos-criterios operativos para definir los empleos informales en función de las circunstancias nacionales y de la información pertinente que pueda recopilarse al respecto. 
c) Sector: comercio, agricultura, industria.

d) Tipo de empresa y tamaño de la misma.

e) Ubicación geográfica: urbana o rural.

f) Protección del empleo: tipo y duración del contrato, derechos laborales.

g) Seguridad social.

\section{SiTUACIÓN LABORAL JUVENIL}

\section{América Latina y el Caribe}

En la región, los jóvenes que logran incorporarse en el mundo laboral lo hacen a través de empleos de poca calidad en donde reciben salarios por debajo de la media, en algunos casos a pesar de contar con competencias superiores a las exigidas para desempeñar el puesto, de tal suerte que su participación tiende a caracterizarse por signos de precariedad — inestabilidad y desprotección-, ya que las instituciones de mercado y la protección social son débiles. ${ }^{22}$

Otro grupo juvenil importante se inserta en la "economía informal", la cual gana cada vez más terreno en este sector de la población: seis de cada diez jóvenes lo hacen en este campo. ${ }^{23}$ Tal situación es particularmente grave porque influye, a futuro, en las trayectorias laborales y personales de los jóvenes y porque da cuenta, por parte de la economía, de la creación de empleos a un ritmo más lento que el crecimiento de la población en edad de trabajar.

En materia de desempleo juvenil, América Latina y el Caribe registró en 2011 una tasa de $13.9 \%$, lo que triplica la tasa correspondiente a los adultos. Entre los países que destacan con mayor desempleo de los jóvenes se encuentran: Guatemala, Honduras, Paraguay y Perú. ${ }^{24}$

Adicionalmente, los países de la región también enfrentan una tasa alta de jóvenes que no estudian ni trabajan, los cuales están mayormente expuestos a ser excluidos tanto del trabajo como de la sociedad. Estos grupos catalogados por su condición de actividad representan una parte dentro de las múltiples juventudes existentes, dados los altos niveles de heterogeneidad de este gru-

22 OIT, Un buen comienæo: trabajo decente para los jóvenes, Ginebra, 2004.

23 Ibidem, p. 9.

24 OIT, Panorama laboral, Ginebra, 2013, p. 70. 
Esta revista forma parte del acervo de la Biblioteca Jurídica Virtual del Instituto de Investigaciones Jurídicas de la UNAM

po social. De acuerdo con la OIT, el grupo incluye a los que no trabajan ni estudian pero buscan empleo, también a los jóvenes —en su mayoría mujeres- dedicadas a los quehaceres del hogar y a otros inactivos, principalmente hombres, quienes no estudian, no trabajan, ni buscan empleo y tampoco se dedican a labores domésticas, es decir, no realizan ninguna actividad por lo que se les reconoce como el "núcleo duro" (4.6 millones). ${ }^{25}$ Se estima que cerca de 21.8 millones conforman este grupo y representan el $20.3 \%$ del universo juvenil en la región.

\section{México}

El país también encara las dificultades y necesidades de los diferentes colectivos juveniles que constituyen los grupos críticos que conforman las nuevas dinámicas sociales: los ocupados, los desempleados y los que no estudian ni trabajan.

En 2013, de 21.5 millones de jóvenes ${ }^{26}$ que representaban el 18.2\% de la población total, ${ }^{27}$ se ubicaban en el mundo laboral sólo 9.1 millones (90.8\%) en las siguientes condiciones: el 77.4\% realizaba un trabajo subordinado, el $8.2 \%$ tenía un trabajo independiente o por su cuenta y el $14.4 \%$ no percibía remuneración alguna (quizá se encuentran en los denominados aprendices o practicantes profesionales).

Las unidades económicas en donde se insertaron fueron: en negocios familiares registrados el 28\%; en empresas constituidas el $27.3 \%$; en negocios de pequeña escala no registrados — de manera independiente o subordinada- el 26.8\%; en la agricultura un 6.4\%; en la administración pública el $5 \%$; en el servicio doméstico remunerado el 3.7\%; en instituciones privadas —asociaciones civiles - el 1.5\% y el resto no se pudo identificar (1.3). ${ }^{28} \mathrm{Como}$ puede apreciarse, se insertan en ramas de actividad y en empresas asociadas

25 OIT, Trabajo decente y juventud..., cit., p. 42.

26 Dado que en México la población juvenil comprende de 12 a 29 años, en 2014 este sector de la población asciende a más de 37 millones, representando el $32.1 \%$ del total de la población. Sin embargo, el INEGI presenta información sociodemográfica acerca de la población de 15 a 29 años que reside en el país y para el segundo trimestre del 2014 reportó 14 millones de jóvenes ocupados.

27 INEGI, Panorámica de la población joven en México desde la perspectiva de su condición de actividad 2013, México, INEGI, 2014.

28 INEGI, Encuesta Nacional de Ocupación y Empleo, II trimestre de 2013. 
Esta revista forma parte del acervo de la Biblioteca Jurídica Virtual del Instituto de Investigaciones Jurídicas de la UNAM

a sectores de baja productividad, con mínimas remuneraciones (en algunos casos, a pesar de contar con competencias superiores a las exigidas para desempeñar el puesto)..$^{29}$ Adicionalmente, en las reestructuraciones son el grupo más perjudicado debido al tipo de contratos, ocupaciones y sectores en los que trabajan. A menudo, compiten con trabajadores con más experiencia y plenamente formados.

Por otro lado, 5.8 millones se encontraban insertos en negocios no registrados y empresas formales sin acceso a prestaciones de seguridad social, esto es, el 63.8\% del total de este grupo social se ubicaba en la "economía informal", preferentemente un mayor número de mujeres así como aquéllos que se ubican en el quintil de ingresos más bajos, con inferiores grados de escolaridad (primaria incompleta el $90.3 \%$ o completa el $84.3 \%$ ). También fue posible encontrar en este rubro, a aquéllos con estudios de medio superior y superior (un 44\%). ${ }^{30}$ Dentro de este grupo, se distinguían tres categorías: a) los que percibían hasta un salario mínimo $(17 \%)$; b) los que reciben más de uno y hasta dos salarios mínimos, uno de cada tres: $32.2 \%$, y c) los que no reciben remuneración, uno de cada seis: $17.1 \%$. De los puestos de trabajo que se crean cada año sólo una cuarta parte fue para los jóvenes.

Igualmente, en 2013 en el desempleo juvenil se ubicaron 923,000 (9.2\%), de los cuales un porcentaje considerable carecía de experiencia laboral. ${ }^{31} \mathrm{De}$ esta forma, el desempleo del grupo, en ese año fue doble con relación al del adulto que representó el 4.2\%, lo que refleja la disparidad de oportunidades para el grupo juvenil. ${ }^{32}$ Gran parte de los desocupados (41\%) ${ }^{33}$ tiene estudios de nivel medio superior y superior, y cada año se suman al mercado laboral más de 450 mil estudiantes que concluyen sus estudios, pero al no existir la creación de empleos suficientes para atender esta demanda, se acentúa el rezago laboral principalmente en las entidades con mayor oferta educativa.

Algunas entidades federativas presentan mayores tasas de desempleo juvenil debidas en parte a que no sólo afrontan su propia problemática de em-

29 Entre más elevado es el grado de estudios menos son las probabilidades de encontrar empleo acorde a su formación profesional.

30 INEGI, Estadísticas a propósito del Día Internacional de la Juventud, agosto de 2014.

31 De acuerdo con el INEGI el $18.7 \%$ de los jóvenes desocupados no cuentan con experiencia laboral.

32 El desempleo juvenil afecta de modo similar a hombres y mujeres, aunque la tasa de estas últimas es mayor.

33 INEGI, Encuesta Nacional de Ocupación y Empleo, II trimestre de 2014. 
Esta revista forma parte del acervo de la Biblioteca Jurídica Virtual del Instituto de Investigaciones Jurídicas de la UNAM

pleabilidad, sino que se suma a ello el atender a jóvenes provenientes de otros estados del país. ${ }^{34}$ Sin embargo, en aquellos estados con tasas bajas de desempleo no necesariamente significa que haya generación de empleos, ya que la experiencia internacional muestra que ante tasas bajas de desempleo juvenil se oculta, posiblemente, un alto porcentaje de trabajo en la "economía informal", o bien el desplazamiento de los jóvenes a otras entidades en busca de trabajo. ${ }^{35}$

Dentro de los que no estudian ni trabajan 1.2 millones se ubicaron en alguna actividad que no implicaba remuneración alguna, tal como: tareas del hogar, cuidados a terceros, estudiar, mantenimiento de la vivienda y de las posesiones del hogar, realizar compras y trámites, así como la prestación de servicios bajo la modalidad de trabajo voluntario (1.3 millones) ${ }^{36}$ y 2.2 millones efectivamente no asistían a la escuela ni estaban interesados en incorporarse al mundo del trabajo.

El panorama laboral de los jóvenes mexicanos muestra que éstos están muy alejados de un trabajo decente. ${ }^{37}$ Gran parte de las dificultades en la incorporación laboral digna o decente se debe, principalmente, a un insuficiente crecimiento económico y una baja productividad que permita la creación de mejores empleos; asimismo, a diversos obstáculos: baja escolaridad, desvinculación del sistema educativo con el productivo, alta proporción de jóvenes que no estudian ni trabajan, elevadas tasas de desempleo de este sector, entre otros.

34 Como lo establece la Encuesta Nacional de Ocupación y Empleo (ENOE), correspondiente al segundo trimestre de 2014, las entidades federativas con mayores tasas son: Distrito Federal, Estado de México (11.3\%), Durango (11.3), Tabasco (11.1\%) y Aguascalientes (10.8).

35 Según la ENOE II, 2014, las entidades con menores tasas son: Guerrero (2.7), Oaxaca (4.1\%), Yucatán (4.4\%), Chihuahua (5.3\%) y Michoacán (5.4\%). Sin embargo, se confirma la regla de que registran altas tasas de empleo informal, por ejemplo en 2013: Oaxaca, Guerrero y Michoacán con el $81.2 \%, 80 \%$ y $72 \%$, respectivamente. Cfr. Nuevas estadísticas de informalidad laboral.

36 El INEGI señala que tradicionalmente tiende a clasificarse la población en términos de la relación que guardan con la actividad económica, a través de los conceptos de población económicamente activa y no económicamente activa, sin embargo, este esquema clásico puede intersectar con otro, en el cual la persona está o no ocupada en el mercado laboral pero realiza otras cosas fuera de él, que no suponen remuneración alguna. Generalmente, se trata de las mujeres cuya tasa sobrepasa a la de los hombres.

37 Aquel "productivo con remuneración justa, seguridad en el lugar de trabajo y protección social para el trabajador y su familia, mejores perspectivas para el desarrollo personal y social, libertad para manifestar sus preocupaciones, se organicen en la toma de decisiones que afectan a sus vidas, así como la igualdad de oportunidades y de trato para mujeres y hombres". 
Esta revista forma parte del acervo de la Biblioteca Jurídica Virtual del Instituto de Investigaciones Jurídicas de la UNAM

Por el lado del crecimiento económico, puede decirse que en la primera década del presente siglo su desempeño ha sido inferior al $4.5 \%,{ }^{38}$ porcentaje considerado como el punto de inflexión para que el mercado demande mano de obra juvenil (se estima que en este año y el siguiente podría crecer a un $1.2 \%$ y $3.5 \%$ ); por su parte, la baja productividad impulsa la creación de empleos precarios.

Por el lado de los obstáculos, como lo afirma la Organización Internacional del Trabajo, ${ }^{39}$ los jóvenes enfrentan un desajuste de las competencias laborales que pone a la vista las inconsistencias entre los sistemas educativos y la demanda laboral: a) una sub-educación y escasez de competencias para el trabajo, y b) la coexistencia de una sobre-educación y exceso de competencias. ${ }^{40}$ La falta de empleos de calidad ha generado un desequilibrio entre la oferta y demanda de profesionistas, lo cual repercute en altas tasas de desempleo en los niveles educativos más altos y se convierte en un problema creciente porque la oferta de jóvenes capacitados supera la capacidad de las economías para los empleos que ellos necesitan. ${ }^{41}$

En resumen, los jóvenes se encuentran entre la precariedad ${ }^{42}$ — con mayor fuerza en América Latina y el Caribe y en el caso nacional—, porque los que tienen empleo lo realizan con un alto grado de inobservancia de las leyes laboral y de seguridad social. Aquellos que laboran por su cuenta sufren los inconvenientes de la desprotección y de la incertidumbre de los ingresos.

Igualmente, el grupo juvenil es especialmente vulnerable a los déficits más graves de trabajo decente, sobre todo en la economía informal. ${ }^{43}$ Pesa sobre

38 Rodríguez, Laura, Políticas públicas para promover el empleo juvenil y el emprendedurismo de los jóvenes en México, México, 2010, p. 10.

39 OIT, Tendencias mundiales del empleo juvenil 2013, Ginebra, 2013, pp. 24-38.

40 Sólo entre el 20\% y el 25\% de la población juvenil ocupada percibe que existe una correspondencia entre su nivel de escolaridad y el trabajo desempeñado.

41 En México el 38\% de los jóvenes egresados del nivel universitario no se desempeñan en las actividades para los cuales fueron formados (cuatro de cada diez) y el 15\% se encuentran desempleados.

42 La OIT concibe al empleo precario como la relación laboral donde falta la seguridad, uno de los elementos principales del contrato de trabajo, término que comprende al temporal, ocasional, a corto plazo, estacional, a tiempo fijo, trabajo a domicilio y la subcontratación. Desde una perspectiva jurídica es lo opuesto al empleo tradicional, y en forma concreta hace referencia a la pérdida de los derechos contractuales, jurídicos-laborales y normas protectoras.

$43 \mathrm{Al}$ igual que las mujeres, los migrantes, los trabajadores de edad, los pueblos indígenas y tribales, las personas afectadas por el sida y las personas con discapacidad. 
Esta revista forma parte del acervo de la Biblioteca Jurídica Virtual del Instituto de Investigaciones Jurídicas de la UNAM

él un conjunto de factores que afectan de muy diversas maneras su sano desarrollo, en el orden físico, mental y profesional, entre ellos, sus antecedentes económicos, bajo nivel de educación, falta de competencias, género, etcétera. Este panorama muestra que la inserción laboral de los jóvenes en un trabajo decente es un objetivo difícil de alcanzar.

\section{ESTRATEGIAS INTERNACIONALES}

La situación del empleo juvenil a nivel internacional confirma que el desafío del déficit del trabajo decente para este sector es de gran magnitud y de una amplia heterogeneidad. Al respecto, en los diversos documentos de la Organización Internacional del Trabajo y, en específico, la resolución de 2005 “La crisis del empleo de los jóvenes: ¡Actuemos ya!” y la del 2012 “La crisis del empleo juvenil: un llamado a la acción", ${ }^{44}$ se pugna por el desarrollo de políticas diferenciadas para atender la problemática juvenil, porque se concluye que no existen soluciones simples y masivas para revertir las situaciones tan complejas y estructurales, y porque si bien, el crecimiento económico es esencial para generar más empleos de mejor calidad, no es suficiente sino va acompañado de políticas sociolaborales complementarias como las siguientes: ${ }^{45}$

a) A nivel macro, consustanciales a cualquier política de empleo. Macro y microeconómicas que impulsen la empleabilidad y eleven oportunidades de empleo productivo; macroeconómicas favorables al empleo que refuercen la demanda agregada y mejoren el acceso a la financiación; medidas que promuevan el crecimiento sostenible del sector privado y de las empresas, políticas anticíclicas y programas públicos de empleo, mecanismos de garantía de empleo, subsidios salariales, formación y políticas orientadas a la transición al empleo formal.

b) Focalizadas dirigidas a los diferentes colectivos juveniles (ocupados en la economía informal, desempleados e inactivos). Programas de incentivación a la formalización, piso básico de protección social, empleabilidad, educación, formación y competencia, iniciativa empresarial, programas de inserción

44 OTI, Resolución relativa al empleo de los jóvenes: vias para acceder a un trabajo decente, Ginebra, 2005; La crisis del empleo juvenil: un llamado a la acción, Ginebra, 2012.

45 OIT, Trabajo decente y juventud..., cit., p. 81. 
laboral: pasantías, aprendizaje, primer empleo, programas de segunda oportunidad, programas de participación juvenil.

c) Centradas en las condiciones efectivas para el acceso y ejercicio de los derechos fundamentales en el trabajo. Políticas de empleo en consonancia con las obligaciones nacionales y en el marco de las normas internacionales del trabajo, protección de los derechos de sindicalización y negociación colectiva, cumplimiento de salarios mínimos aplicables a este sector, mayor participación y representación juvenil en las organización para alentar el diálogo social.

Como puede apreciarse, cada grupo de las políticas diferenciadas amerita un estudio particular, sin embargo, dada la magnitud del empleo en la "economía informal" a nivel regional y nacional así como su importancia en el debate laboral con miras al establecimiento de una recomendación para la transición hacia la formalidad, nos enfocaremos a este tópico con atención a los jóvenes.

Desde el 2002, la OIT ha abordado este problema. Primero, en el Informe VI de la 90a. reunión de la Conferencia Internacional del Trabajo y en las conclusiones sobre el trabajo decente y la economía informal. ${ }^{46}$ Asimismo, en 2007 retomó el asunto en el Coloquio interregional tripartito "Hacer posible la transición al sector formal”, en el cual se examinaron una serie de buenas prácticas y enfoques innovadores desarrollados por los gobiernos, los interlocutores sociales y otros actores.

A nivel internacional se asume que el tránsito a la formalidad trae consigo beneficios tangibles en áreas tales como la pobreza, la desigualdad, la productividad y el apoyo a la democracia. De igual forma, permite beneficios palpables en los ingresos de los jóvenes trabajadores en esta situación

En razón de lo anterior y con base en estos trabajos, la OIT considera que se requiere un enfoque global e integrado para frenar la expansión de la "economía informal”. ${ }^{47}$ Sugiere siete vías para lograrlo: 1) generación de empleos de calidad y estrategias de crecimiento; 2) entorno normativo: observación de las normas internacionales de trabajo y los derechos fundamentales; 3) diálogo social, organización y representación; 4) fomento a la igualdad y lucha contra

46 OIT, El trabajo decente y la economía informal, Informe VI, CIT, 90 reunión, Ginebra, 2002, pp. $50-53$.

47 Hasta ahora las políticas seguidas por los países de América Latina han sido puntuales, descoordinadas, ad hoc o limitadas a ciertas categorías de trabajadores. 
Esta revista forma parte del acervo de la Biblioteca Jurídica Virtual del Instituto de Investigaciones Jurídicas de la UNAM

la discriminación; 5) medidas de apoyo a la iniciativa empresarial, competencias profesionales y financiación; 6) ampliación de la protección social: establecimientos de pisos de protección social y sistemas de seguridad social, y 7) estrategias de desarrollo local (rural y urbano).48

De estas vías se recomiendan como intervenciones específicas para atender el empleo juvenil en la economía informal: programas de incentivos a la formalización, piso básico de protección social, educación, formación y competencias, iniciativa empresarial y empleo por cuenta propia, ${ }^{49}$ las cuales a continuación se analizarán.

\section{Programas de incentivos a la formalización}

Facilitar la transición a la formalidad implica, primero, reconocer la heterogeneidad de la economía informal, sus diferentes categorías y los factores que dan lugar a su crecimiento y, segundo, aplicar un enfoque integrado de políticas sobre productividad, normas, incentivos y fiscalización para abordar el problema en sus distintas dimensiones, diferentes actores y colectivos. ${ }^{50}$

La reducción de este fenómeno es un componente esencial para disminuir la desigualdad y la exclusión social, pero a la vez se tiene que velar porque las oportunidades, la subsistencia y la capacidad empresarial no se destruyan. En las políticas de formalización se tienen que distinguir entre los trabajadores independientes en empresas informales y los empleadores que evaden el cumplimiento de las leyes laborales y de seguridad social.

De acuerdo con la OIT los trabajadores y las unidades económicas de la economía informal pueden tener un gran potencial empresarial, y su creatividad, dinamismo, competencias y capacidad de innovación pueden desarrollarse al eliminar los obstáculos hacia su gradual transición a la economía formal.51

Dentro del enfoque integrado, la OIT sugiere facilitar una transición gradual, a través de enfoques específicos dada la diversidad de circunstancias de los trabajadores y de las unidades económicas de la economía informal. Un

\footnotetext{
48 OIT, La transición de la economía informal..., cit., V (1).

49 OIT, Trabajo decente y juventud..., cit., p. 81.

50 OIT, Experiencias recientes de formalización en países de América Latina y el Caribe, Oficina Regional para América Latina y el Caribe, Forlac, 2014, p. 8.

51 OIT, La transición de la economía informal...., cit., V (2), p. 88.
} 
Esta revista forma parte del acervo de la Biblioteca Jurídica Virtual del Instituto de Investigaciones Jurídicas de la UNAM

enfoque equilibrado que combine incentivos y medidas destinadas a promover su cumplimiento.

En este sentido, en 2013 la Oficina Regional de la OIT para América Latina y el Caribe puso en marcha un ambicioso programa de formalización de la informalidad, denominado FORLAC. El programa tiene tres componentes: a) generación y diseminación de conocimiento en torno las políticas de formalización; b) asistencia técnica a países específicos, y c) fortalecimiento de trabajadores y empleadores en formalización.

Con base en dicho programa, la OIT recomienda adoptar medidas para la transición gradual de los trabajadores a la economía formal a través de:

a) Simplificar el registro de empresas y el cumplimiento de las obligaciones tributarias.

b) Asegurar el otorgamiento de beneficios, tales como: acceso a servicios, financiamiento, seguros, infraestructura, mercados, tecnología, programas de capacitación y derechos de propiedad, información de los diversos reglamentos y oportunidades del mercado con miras a mejorar la productividad, el desarrollo de la capacidad empresarial, la gestión personal, la contabilidad y otros aspectos.

c) Extender la seguridad social, a través de adaptar los procedimientos, las prestaciones y las cotizaciones, de acuerdo a la capacidad contributiva de los sujetos, la prestación de servicios de guardería y otros servicios de cuidado de calidad que sean asequibles a fin de promover las oportunidades de empleo de las mujeres.

d) Respecto de las pequeñas y medianas empresas asegurar que los bancos e instituciones de fomento les brinden recursos a fin de que cumplan un rol activo para mantener su liquidez, sobre todo, al enfrentar un contexto crediticio más restrictivo. ${ }^{52}$

\section{Piso básico de protección social}

De acuerdo con la OIT, con el objeto de contribuir eficazmente al proceso de transición al empleo formal, es necesario proporcionar garantías de protección social básica en el marco de las políticas públicas generales, integrales

52 OIT, El empleo informal en México..., cit. 
Esta revista forma parte del acervo de la Biblioteca Jurídica Virtual del Instituto de Investigaciones Jurídicas de la UNAM

y coherentes, basadas en una coordinación eficaz entre las políticas estatales en los ámbitos de educación, salud, seguridad social, empleo y las cuestiones laborales, así como las políticas económicas y fiscales. Se sabe que la falta de protección social es un aspecto clave de la exclusión social de los jóvenes en la economía informal. Éstos, al igual que los demás grupos de edad, necesitan tener acceso a una gama de prestaciones sociales básicas que les ofrezcan protección contra varias contingencias vitales, sociales y de la vida laboral, con lo cual no sólo se ayudará a hacer realidad la transición a la formalidad, sino también a generar ingresos y aumentar la productividad y la prosperidad..$^{53}$

En concordancia con ello, en 2008 la OIT, en su 97a. reunión, realizó la "Declaración sobre la justicia social para una globalización equitativa", en donde reafirmó el compromiso de ampliar la seguridad social a quienes necesiten protección en el marco del Programa de Trabajo Decente. ${ }^{54}$ Asimismo, la "Recomendación sobre los pisos de protección social de 2012, reconoció que el establecimiento de un sistema de seguridad social sostenible es un importante factor que contribuye a la transición hacia el empleo formal.

Estas propuestas se enmarcan en lo que se ha denominado "la estrategia bidimensional" para la extensión de la cobertura de seguridad social eficaz: 55 a) la dimensión horizontal que busca extender un conjunto mínimo de transferencias, derechos y beneficios, y b) la dimensión vertical consistente en aumentar el alcance de la cobertura y las prestaciones, con la intención de proporcionar mayores niveles de seguridad social a la gente, sobre las políticas destinadas a formalizar gradualmente las economías informales. Estas dimensiones deben estar coordinadas con políticas fiscales, económicas y de empleo. En forma específica, debe garantizarse a los jóvenes:

a) Atención médica, a través de impuestos o subsidios a las cotizaciones.

b) Seguridad de los ingresos, a través de mecanismos de transferencias de ingresos condicionados, para eliminar eventuales incentivos a la permanencia en la informalidad y lograr su tránsito hacia empleos productivos.

53 OIT, La transición de la economía informal..., cit., V (1), pp. 28-54.

54 Estas garantías de seguridad social se enmarcan dentro de la conceptualización del denominado "piso de protección social” iniciativa encabezada por la Organización de Naciones Unidas, la Organización Internacional del Trabajo y la Organización Mundial de la Salud.

55 Cfr. Cichon, Michael et al., La iniciativa del piso de protección social de las Naciones Unidas, cambiando la tendencia en la conferencia de la OIT de 2011, Berlín, Friedrich-Ebert-Stiftung, enero de 2011, p. 9, disponible en: http:/ / library.fes.de/pdf-files/iez/07997.pdf. 
Esta revista forma parte del acervo de la Biblioteca Jurídica Virtual del Instituto de Investigaciones Jurídicas de la UNAM

Igualmente, crear garantías de empleo para los jóvenes con problemas de acceso al mercado de trabajo, esquemas alternativos de cotizaciones bajas o subsidio estatal ${ }^{56} \mathrm{y}$ un seguro de desempleo que proteja y fomente la búsqueda de empleo.

En forma particular, para las mujeres jóvenes, que en la actualidad se ven impedidas en acceder al mercado de trabajo, continuar sus estudios, a causa de embarazos, se recomiendan medidas de diferentes niveles: información para paliar los embarazos adolescentes, medidas para evitar que el cuidado de los hijos no conduzca al abandono escolar o la exclusión de una trayectoria laboral positiva y medidas de compensación que permitan la construcción de un sistema de economía del cuidado.

\section{Educación, formación y competencias}

La OIT da cuenta de que se accede a la economía informal, por un lado, porque los niveles en materia de educación, calificaciones, tecnología y capital exigidos en dicha economía son muy bajos y, por otro, porque los jóvenes necesitan contar con ingresos básicos. En el empleo, en esta economía, es precisamente en donde hay mayor insuficiencia de competencias profesionales, lo cual se traduce en un círculo vicioso de bajos niveles de productividad, ingresos e inversión. Así, mejorar las competencias profesionales de los jóvenes en la economía informal es un elemento estratégico para aumentar la empleabilidad de éstos, la productividad de las empresas, aliviar la pobreza y ayudarlos a acceder a empleos remunerados y productivos en la economía formal. ${ }^{57}$

De acuerdo con la OIT,

...gracias a la educación es posible mejorar las condiciones sociales, económicas y culturales de los países. El aumento de los niveles educativos tiene efectos positivos sobre factores claves de desarrollo y bienestar, como la

56 OIT, Piso de protección social para una globalización equitativa e inclusiva, Ginebra, 2010; Can Low Income Countries Afford Basic Social Security? Social Security Policy Briefing, Ginebra, Oficina Internacional del Trabajo, 2008, disponible en: http:// mmw.ilo.org/public/english/protection/secsoc/ downloads/policy/policy3e.pdf.; Trabajo decente y juventud en América Latina, Lima, 2013; La transición de la economía informal a la economía formal, Conferencia Internacional del Trabajo, 103a., reunión, 2014.

57 OIT, La transición de la economía informal..., cit., V (1), p. 47. 
Esta revista forma parte del acervo de la Biblioteca Jurídica Virtual del Instituto de Investigaciones Jurídicas de la UNAM

productividad, el ingreso, el empleo y la competitividad, además de la construcción de la ciudadanía, la identidad social y el fortalecimiento de la cohesión social..$^{58}$

Por ello, reconoce la importancia de los sistemas de educación como factores clave que determinan los resultados del empleo juvenil al ofrecer habilidades y aptitudes necesarias para desenvolverse en el mundo laboral y, por consiguiente, para facilitar la transición de la escuela al trabajo.

Dados los cuestionamientos tanto a la educación formal, a la educación técnica y la formación profesional, surgió en el escenario internacional el concepto de "competencias" en sintonía con la Resolución 195 de la OIT "Sobre el desarrollo de los recursos humanos", 59 con las que se pretende una necesaria articulación entre las demandadas por el mercado y las adquiridas en la educación, para asegurar un impacto positivo en los niveles de bienestar de las personas y en los niveles de productividad en las empresas. ${ }^{60}$

Para la OIT, el desarrollo de las competencias es un factor esencial para alcanzar el objetivo del trabajo decente y productivo, en condiciones de libertad, equidad, seguridad y dignidad humana, que sintetiza los cuatro objetivos estratégicos de dicha organización: oportunidades de empleo e ingresos; protección y seguridad social; diálogo social y tripartismo, y normas y principios fundamentales en el trabajo.

En el ámbito educativo, las competencias implican que el proceso de enseñanza y el aprendizaje no debe circunscribirse exclusivamente a la mera adquisición de conocimientos sino incluir los tres saberes: el saber ser, el saber conocer y el saber hacer; y en el ámbito laboral, la expresión se liga a la inserción en el empleo.

En este sentido, el concepto de competencias, por un lado, es asumido como una estrategia que retoma de manera integral el proceso de enseñanza y aprendizaje, o bien como un modelo educativo globalizado, y por otro lado, como nuevas habilidades para nuevos empleos. Es por ello, que el término

58 OIT, Trabajo decente y juventud..., cit., p. 16.

59 OIT, Recomendación sobre el desarrollo de los recursos bumanos: educación, formación y aprendizaje permanente, adoptada en la 92a. reunión de la Conferencia Internacional del Trabajo, Ginebra, 2004.

60 Vargas, Fernando, "Relación educación y empleo", ponencia presentada en el Foro Internacional de Políticas Públicas de Empleo y Protección Social, México, organizado por la UNAM, OIT, y la Secretaría del Trabajo y Previsión Social, noviembre de 2013. 
ha sido recurrente tanto desde la perspectiva educativa como la laboral, como dan cuenta desde hace varios años organismos internacionales como la UNESCO, la OIT y la Organización para la Cooperación y el Desarrollo Económico (OCDE). ${ }^{61}$

\section{A. Competencias básicas}

Son fundamentales para la vida en sociedad y en las cuales se encuentran las capacidades mínimas necesarias para aprender una profesión y conforman la base sobre la que se forman las competencias restantes. Comprenden las nociones en lectura, escritura y aritmética necesarias como prerrequisito para proseguir la educación, un aprendizaje continuo y conseguir trabajo. ${ }^{62}$

\section{B. Competencias transferibles}

Son comunes a varias ocupaciones o profesiones y refieren la capacidad de afrontar cambios continuos, a la adaptabilidad en diversos contextos laborales en la práctica profesional y/o laboral. Se dividen en:

a) Aprender a aprender. Conocimientos, actitudes y aptitudes para establecer, planear y alcanzar sus metas de aprendizaje, ser independientes y aprender de forma autónoma.

b) Comunicación. Capacidad de comprender a los demás: escuchar, leer y observar usando medios formales e informales —escritos y orales-, y transmitir ideas de manera clara y efectiva.

c) Trabajo en equipo. Capacidad para operar de manera eficiente y efectiva en un grupo desde el punto de vista de la cooperación y del liderazgo.

d) Solución de problemas. Evaluar información y situaciones, y decidir los métodos más apropiados de solución: toma de conciencia de las consecuencias a largo plazo de las acciones y la capacidad de evaluar y adaptar planes para la acción.

61 La OCDE afirmó en varios documentos que el problema de desempleo tiene que ver con la capacidad de innovación, adaptación y aprendizaje continuo; características relevantes dentro de la cultura de las competencias vinculadas a los estadios tanto académicos como laborales actuales.

62 Existen más de ciento treinta millones de jóvenes que carecen de las capacidades básicas. 
Esta revista forma parte del acervo de la Biblioteca Jurídica Virtual del Instituto de Investigaciones Jurídicas de la UNAM

\section{Competencias técnicas y específicas}

Son propias de cada ocupación o profesión. Refiere destrezas que se requieren en las áreas ocupacionales relevantes a la demanda del mercado de trabajo y para una buena inserción laboral. ${ }^{63}$

\section{Iniciativa empresarial de los jóvenes}

La OIT ve la iniciativa empresarial como un medio para crear un trabajo decente y productivo, y para el caso de los jóvenes el desarrollo de habilidades para los negocios puede contribuir a su capacidad de acceso a las oportunidades de empleo. Saber de negocios es comprender el rol de éstos en la sociedad, así como el importante papel que desempeñan las empresas en la consecución de un desarrollo social, económico y medioambiental sostenible.

En un entorno empresarial idóneo, un joven con una mentalidad emprendedora y una serie de competencias y conocimientos básicos en la materia, puede hacer mucho más que subsistir. ${ }^{64}$

Para el desarrollo de esta iniciativa se recomienda eliminar las fuertes barreras de acceso a los servicios empresariales que afectan singularmente a los jóvenes, y en especial, a los más pobres de este sector, pues el éxito de la iniciativa depende de una amplia gama de medidas para que los jóvenes creen, crezcan y mantengan sus empresas:

a) Facilitar el acceso a fuentes de financiamiento como los microcréditos ${ }^{65}$ (que exigen menos requisitos de capital, pocas garantías o ninguna y experiencia empresarial) o la provisión de préstamos sin intereses o a uno muy bajo, acceso a tecnologías, mercados locales e internacionales.

b) Proporcionar distintos tipos de formación y capacitación, asistencia técnica y tutoría para la elaboración, desarrollo y acompañamiento de un plan empresarial.

63 La OIT al mencionar las competencias laborales las divide en: competencias básicas y las competencias técnico-específicas.

64 OIT, Guía de introducción para las organizaciones de empleadores. Combatir los desafios del empleo de los jóvenes, Centro Internacional de Formación, 2012, pp. 31, 32 y 46.

65 Los microcréditos son pequeños préstamos ofrecidos por instituciones de microfinanciación, como cooperativas de crédito y organizaciones no gubernamentales. 
c) Aumentar el abanico de servicios de apoyo (publicidad, cadenas de producción, distribución, exportaciones, contrataciones públicas).

d) Promover redes de empresarios jóvenes.

e) Apoyar con medidas para aumentar la eficacia de las empresas y potenciar su capacidad para innovar, así como intervenciones para incrementar la productividad y mejorar las condiciones de trabajo.

f) Facilitar la transición de los jóvenes empresarios de la economía informal a la formal ${ }^{66}$ y promover el cumplimiento de la legislación laboral.

g) Apoyos fiscales y en materia de seguridad social. La exención fiscal o los impuestos diferenciados pueden facilitar el proceso de puesta en marcha de una empresa para los jóvenes.

h) Proporcionar acceso fácil a servicios de desarrollo empresarial, como instalaciones para viveros ${ }^{67}$ de empresas.

i) Promulgar normas que favorezcan la creación de microempresas, pequeñas y medianas empresas, cooperativas y empresas sociales sostenibles.

\section{INTERVENCIONES NACIONALES}

\section{Iniciativas desarrolladas}

En las dos últimas décadas se han realizado, en el país, un conjunto variado de intervenciones — políticas y programas - para enfrentar la problemática del empleo juvenil. En algunos casos, las iniciativas son el resultado de revisiones de programas preexistentes o nuevas ediciones, pero se ubican dentro de las acciones recomendadas internacionalmente. ${ }^{68}$

En este marco, las políticas activas de mercado han sido las más recurrentes, mas han sido frecuentes las políticas e instituciones para promover la

66 Principalmente a aquellos situados fuera de la protección de la seguridad social, pero también la formalización de pequeñas y medianas empresas, en un entorno propicio para su desarrollo sostenible, así como la de los jóvenes que trabajan por cuenta propia en condiciones de informalidad.

67 Los viveros de empresas se han convertido en un poderoso recurso para respaldar el proceso empresarial y ayudar a aumentar la tasa de supervivencia de las empresas innovadoras recién creadas por jóvenes.

68 Algunas de las iniciativas se concentran en jóvenes en riesgo y en muchos casos refuerzan sus acciones con condicionalidades en logros escolares. 
Esta revista forma parte del acervo de la Biblioteca Jurídica Virtual del Instituto de Investigaciones Jurídicas de la UNAM

inserción laboral de los jóvenes: subsidios a la contratación, ley del Primer Empleo, ${ }^{69}$ protección social, el Programa Oportunidades que otorgaba transferencias condicionadas para la inserción y retención escolar, complementado con el Programa Jóvenes con oportunidades, ${ }^{70}$ fomento del emprendimiento "Emprendedores Juveniles", ${ }^{71}$ servicios de capacitación y empleo para cubrir la brecha de competencias de los jóvenes como las necesidades de empleo; a través del Chambeatón se facilitó el acceso a la información sobre las oportunidades laborales, y en materia de institucionalidad juvenil se cuenta con el Instituto Mexicano de la Juventud (IMJUVE) quien determina el marco de la construcción de iniciativas sectoriales, entre ellas: las de empleo, órgano que tiene la atribución de elaborar en coordinación con las dependencias y las entidades de la administración pública federal, programas y cursos de orientación e información sobre la incorporación laboral, autoempleo, pero en realidad su actuación es marginal en materia de empleo.

A pesar del conjunto de intervenciones desarrolladas no existe información suficiente sobre la evaluación del desempeño, los principales resultados e impacto de los programas, de tal suerte, que no es posible realizar un análisis sobre lo que ha funcionado. Por tanto, se hace necesario la implementación de sistemas de monitoreo y evaluación, incluyendo costo-beneficio para contar con mayores herramientas para enfrentar las desventajas de los jóvenes en el mundo del trabajo.

69 La ley absorbió al Programa del Primer Empleo e imprimió algunas modificaciones. Los subsidios a la contratación de los jóvenes tomaron la forma de exenciones impositivas para las empresas (reducción de los impuestos sobre los ingresos y el IETU de hasta $40 \%$ durante tres años por cada nuevo trabajador joven).

70 El programa comprendía la educación básica y media superior. Establecía como condiciones para los beneficiarios: 1) alcanzar más años de escolaridad que sus padres; 2) graduarse a los quince años de secundaria; 3) que una fracción mayor del global nacional de mujeres pasen al bachillerato; 4) que el 60\% pasen al bachillerato, y 5) que concluyan el nivel medio superior. Así, "Jóvenes con oportunidades" era un premio para el becario por la conclusión de su educación media superior y si continuaba con sus estudios a nivel superior podría cobrarlo en dos partes: 1) la primera, cuando se inscriba en la universidad (2/3 partes del total de su apoyo), y 2) la segunda, al encontrarse en el siguiente año de la misma carrera (1/3 parte del aроуо).

71 Iniciativa que ofrecía capacitación para desarrollar las competencias empresariales, servicios de apoyo (orientación, tutoría asesoramiento técnico y financiero) y acceso a microcréditos. 
Esta revista forma parte del acervo de la Biblioteca Jurídica Virtual del Instituto de Investigaciones Jurídicas de la UNAM

\section{Intervenciones recientes}

Dentro de este tipo de intervenciones y acorde al objetivo del presente estudio, resulta conveniente revisar las iniciativas más importantes en materia de formalización del empleo, que si bien no están dirigidas específicamente a los jóvenes pueden ser comprendidos en ellas.

En julio de 2013 se inició un programa con el que se pretende lograr en todo el país la transición de la informalidad a la formalidad. El Programa para la Formalización del Empleo fue suscrito el 22 de julio de ese año por el gobierno federal, los gobiernos estatales y el Distrito Federal, el Instituto Mexicano del Seguro Social y la Secretaría del Trabajo y Previsión Social. El programa se engloba dentro de las políticas públicas: reforma educativa, laboral y financiera, ${ }^{72}$ y pretende otorgar una serie de beneficios tanto en materia fiscal como de seguridad social.

\section{A. Régimen de incorporación fiscal}

La reforma fiscal estableció el denominado régimen de incorporación fiscal. Así, con el nuevo esquema de tributación pueden incorporarse las personas físicas con poca capacidad económica ${ }^{73}$ y administrativa. Durante dicho periodo tendrán, entre otros beneficios, descuentos en el pago del Impuesto a la Renta durante diez años (100\% el primer año, disminución gradual del 10\% en cada año hasta llegar al 10\% en el décimo año); poder presentar de forma bimestral sus declaraciones - de pago e informativas - para efectos del impuesto sobre la renta y, de esta manera, se espera que logren cumplir adecuadamente con sus obligaciones e incorporarse al régimen general de la ley. ${ }^{74}$ Asimismo, serán sujetos de créditos a través del programa especial de financiamiento de Nacional

72 SHCP, Fomentando la formalidad laboral en todo el territorio nacional, Informe Semanal del Vocero, julio de 2013.

73 Pueden acceder las personas físicas con actividad empresarial con ingresos menores a dos millones de pesos.

74 Las personas que se incorporen a dicho régimen deberán calcular el impuesto al valor agregado de forma bimestral por los periodos comprendidos de enero y febrero; marzo y abril; mayo y junio; julio y agosto; septiembre y octubre; noviembre y diciembre de cada año, y efectuar el pago del impuesto a más tardar el día 17 del mes siguiente al bimestre que corresponda, mediante declaración que presentarán a través de los sistemas que disponga el Servicio de Administración Tributaria en su página de Internet. 
Esta revista forma parte del acervo de la Biblioteca Jurídica Virtual del Instituto de Investigaciones Jurídicas de la UNAM

Financiera así como acceso preferencial a recursos del Instituto Nacional del Emprendedor, dirigidos a micro, pequeñas y medianas empresas, Mypymes.

El 10 de septiembre de 2014 se adicionaron tres facilidades del régimen de incorporación fiscal. ${ }^{75} \mathrm{La}$ primera, los contribuyentes que realicen ventas al público hasta por 100 mil pesos anuales no pagarán impuesto al valor agregado (IVA) ni impuesto especial de producción y servicios (IEPS) en los primeros diez años de esta estrategia. La segunda, los contribuyentes con ingresos de entre 100,000 y 2 millones de pesos anuales no pagarán tampoco IVA ni IEPS, pero sólo en el primer año de incorporación, además de que se les otorgarán descuentos en los siguientes nueve. La tercera establece un régimen más sencillo para calcular sus impuestos. Bastará con que especifiquen dos datos: la actividad a la que se dedican y el monto de sus ingresos para hacerlo. Además, quien se decida a ser formal recibirá en el primer año un descuento de 100\% en el pago del impuesto sobre la renta (ISR). En el segundo será de $90 \%$ y así sucesivamente. ${ }^{76}$

\section{B. Régimen de incorporación a la seguridad social}

A partir del 1o. de julio de 2014 se puso en operación el régimen de incorporación a la seguridad social, 77 con el que se pretende la incorporación voluntaria de las personas informales al régimen obligatorio del IMSS: trabajadores independientes, ${ }^{78}$ patrones — personas físicas — con trabajadores a su servicio, trabajadores que en términos de ley el empleador está obligado a inscribirlos al IMSS. ${ }^{79}$

Entre los beneficios que recibirán se encuentran: a) el otorgamiento de los seguros de ley aplicables a cada categoría, ${ }^{80}$ y b) subsidios consistentes en

75 Diario Oficial de la Federación, Decreto por el que se otorgan beneficios fiscales a quienes tributen en el Régimen de Incorporación Fiscal, 10 de septiembre de 2014.

76 El decreto entrará en vigor el 1o. de enero de 2015.

77 Diario Oficial de la Federación, Decreto por el que se otorgan estímulos para promover la incorporación a la seguridad social, 8 de abril de 2014.

78 Trabajadores en industrias familiares, independientes, ejidatarios, comuneros, colonos y pequeños propietarios así como empleadores (personas físicas con trabajadores a su servicio).

79 Los sujetos del artículo 12 de la Ley del Seguro Social, aquéllos que tienen una relación laboral.

80 Trabajadores independientes: prestaciones en especie de los seguros de enfermedades así como los seguros de invalidez y vida, retiro y vejez. Personas físicas con trabajadores a su servicio: riesgos de trabajo; prestaciones en especie del seguro de enfermedades; invalidez y vida, 
Esta revista forma parte del acervo de la Biblioteca Jurídica Virtual del Instituto de Investigaciones Jurídicas de la UNAM

un porcentaje de descuento sobre las cotizaciones al Seguro Social y al Fondo Nacional de la Vivienda para los Trabajadores (Infonavit), hasta por diez años, en los siguientes términos: ${ }^{81}$

\begin{tabular}{|c|c|c|c|c|c|c|c|c|c|}
\hline Año 1 & Año 2 & Año 3 & Año 4 & Año 5 & Año 6 & Año 7 & Año 8 & Año 9 & Año 10 \\
\hline $50 \%$ & $50 \%$ & $40 \%$ & $40 \%$ & $30 \%$ & $30 \%$ & $20 \%$ & $20 \%$ & $10 \%$ & $10 \%$ \\
\hline
\end{tabular}

Asimismo, tendrán facilidades de pago de las cotizaciones al modificarse los plazos de pago mensual a bimestral en el régimen obligatorio y de anual a bimestral en el aseguramiento voluntario al régimen obligatorio.

Resta decir que los descuentos en las cuotas aplican en función del año en el que los sujetos —objeto del beneficio— - se registren en el régimen de incorporación fiscal.

\section{Programa "Crezcamos Juntos"}

Es una estrategia que conjunta diversos programas federales para promover la transición a la formalidad. Al Programa de Incorporación a la Seguridad Social se suman los créditos para vivienda, apoyo a emprendedores, financiamiento a empresas y créditos al consumo. Estos nuevos beneficios están condicionados a la incorporación al régimen fiscal. ${ }^{82}$

a) Obtener un crédito para vivienda. El contribuyente deberá hacer aportaciones al Infonavit durante cuatro bimestres si tiene patrón o siete si no cuenta con relación laboral, periodos tras lo que podrá acceder a un crédito con tasas bajas. ${ }^{83}$ El crédito puede usarse para refinanciar hipotecas existentes. El gobierno subsidiará parte de los pagos hipotecarios.

retiro y vejez; y trabajadores sujetos del aseguramiento obligatorio, todos los seguros: riesgos de trabajo; enfermedades y maternidad; invalidez y vida; retiro de cesantía en edad avanzada y vejez; guarderías y prestaciones sociales.

81 Se establecen como condiciones que el patrón y sus trabajadores no hayan cotizado al IMSS o aportado al Infonavit, durante alguno de los 24 meses previos a la fecha de solicitud de aplicación del subsidio, salvo que se trate del ex régimen de pequeños contribuyentes (REPECOS) que hayan cumplido a más tardar el 15 de febrero de 2014 con la presentación de la Declaración Informativa ante el SAT que establece el decreto.

82 El programa se dio a conocer el 8 de septiembre de 2014.

83 El crédito se otorgará mediante un programa de la Sociedad Hipotecaria Federal. En una primera etapa se otorgarán 110,000 créditos para adquirir o construir una casa. 
Esta revista forma parte del acervo de la Biblioteca Jurídica Virtual del Instituto de Investigaciones Jurídicas de la UNAM

b) Apoyos para emprendedores. ${ }^{84}$ Acceso a un apoyo económico de 4,000 pesos para mejorar equipo de negocios. Mediante Bansefi se otorgarán 2,000 pesos en una cuenta bancaria de débito. A su vez, se pueden obtener otros 2,000 pesos para invertir.

c) Financiamiento para las empresas. Acceso a créditos de 20,000 pesos hasta 300,000 pesos a través de Nacional Financiera. Los préstamos están dirigidos a negocios con más de dos años de operación salvo aquellos que lleven un año y pidan hasta 50,000 pesos. Los plazos van desde los veinticuatro hasta sesenta meses según el destino de los recursos.

d) Crédito al consumo. Acceso al crédito del Fondo Nacional para el Consumo de los Trabajadores (Fonacot). El dinero puede destinarse a la compra de aparatos electrónicos, servicios turísticos, muebles, atención médica, entre otros. Existen tasas de interés desde el 10\%; no es necesario contar con experiencia crediticia.

\section{Inspección del trabajo}

En esta materia se promulgó un nuevo Reglamento ${ }^{85}$ que se aplicará a 5 millones de unidades económicas registradas por el INEGI en el país, entre las que se encuentran las 849,000 empresas formales registradas en el Seguro Social. El reglamento además de contemplar asesoría y orientación para las empresas, faculta a la autoridad laboral a realizar inspecciones "sorpresa", 86 con carácter preventivo y/o de cumplimiento, ${ }^{87}$ sobre todo, cuando por vía de quejas o denuncias se tenga conocimiento de la existencia de un "peligro o riesgo inminente", posibles violaciones a la Ley Federal del Trabajo, falseamiento de documentación por parte del patrón, entre otros. En tales

84 A través del Instituto Nacional del Emprendedor recibirán los apoyos económicos, más de 120,000 pequeños empresarios.

85 Reglamento General de Inspección del Trabajo y Aplicación de Sanciones publicado el 17 de junio en el Diario Oficial de la Federación, el cual sustituye a aquel que databa de 1998.

86 Se inspeccionará desde grandes empresas hasta tiendas de abarrotes (las empresas de quince trabajadores o menos sólo serán objeto de visitas de asesoría). Lo ideal sería la identificación de empresas outsourcing que evadan sus obligaciones patronales así como detectar las "oficinas virtuales".

87 La Dirección General de Inspección Federal del Trabajo podrá ordenar la práctica de inspecciones extraordinarias a las empresas "en cualquier tiempo, incluso en días y horas inhábiles", sin que medie citatorio previo. 
supuestos, procederá la suspensión de actividades y multas más elevadas. ${ }^{88} \mathrm{El}$ reglamento contempla algunas herramientas para que la autoridad laboral no pase de manera inmediata a la sanción; ${ }^{89}$ en específico, hay etapas para que sobre todo las micro y pequeñas empresas (Mipymes) puedan cumplir.

\section{CONCLUSIONES}

El mercado de trabajo mexicano se caracteriza por un alto empleo informal, el cual afecta principalmente a los jóvenes. Es un hecho que abordar el problema del empleo informal requiere al menos dos dimensiones: a) crecimiento económico para generar empleos formales, y b) incentivar la formalización a través de estrategias sociolaborales.

Existen experiencias nacionales destinadas a reducir este fenómeno, ${ }^{90}$ pero tanto éstas como las recientes iniciativas, son generales, no distinguen entre los jóvenes asalariados en empleos informales y los independientes en empresas informales, cuando son categorías que tienen sus propias necesidades y sobre todo porque más del $60 \%$ de los jóvenes se encuentra en este tipo de empleo.

La iniciativa de formalización del empleo, en el fondo, tiene como ventajas la postergación del pago del impuesto sobre la renta así como el de las aportaciones de seguridad social, porque en realidad los beneficios en el otorgamiento de los seguros ya están considerados en la propia Ley del Seguro Social. De acuerdo con datos del INEGI, el número de empleos formales registrados ante el IMSS en octubre de 2014 fue de 172,000, de los cuales 134 nuevas plazas (sin especificar cuántos corresponden a jóvenes). Asimismo, se señala una reducción de casi tres puntos porcentuales de la tasa de informalidad laboral en los últimos dos años y la realización de más de 60,000 inspec-

88 Las multas son de hasta cinco mil veces el salario mínimo y pueden multiplicarse de acuerdo al número de trabajadores afectados por el incumplimiento del patrón.

89 Se otorga un periodo de entre treinta y noventa días para corregir las fallas.

90 Si bien es cierto que cada entidad federativa del país refleja una situación distinta tanto en niveles como evolución de la informalidad, algunas han realizado acciones para la formalización del empleo: Jornadas de acercamiento laboral específico en Nuevo León; seguro de desempleo, portal del empleo y fomento cooperativo en el Distrito Federal; el Tuzobús en Hidalgo; La Red Q, en Querétaro y de manera general, la inspección laboral focalizada. 
Esta revista forma parte del acervo de la Biblioteca Jurídica Virtual del Instituto de Investigaciones Jurídicas de la UNAM

ciones en esta materia. Se esperaría que la tendencia sea por este camino y que no resulte ser un falso amanecer.

Sin embargo, es necesario insistir en materia de empleo juvenil ante la ausencia de un marco legal integrado en el que exista complementariedad entre las distintas leyes, programas o mecanismos. El tema debe ser un objetivo de la política nacional, sectorial y de desarrollo. Por ello, es indispensable contar con un Plan de Acción Nacional de Empleo Juvenil, instrumento necesario para consolidar institucionalmente las políticas e intervenciones de promoción del empleo de los jóvenes a nivel nacional, así como para mejorar la coordinación e integración a fin de disminuir la dispersión de esfuerzos y la duplicidad de acciones. ${ }^{91}$ Por tanto, se requiere realizar esfuerzos sustantivos para articular diferentes acciones bajo un enfoque integrado y completo que a corto, mediano y largo plazo enfrente el reto del empleo juvenil.

Entre ello, deberá incluir la legislación federal que atienda las problemáticas juveniles y la regulación jurídica para fomentar el empleo de este sector de la población — completar la reforma al marco jurídico en la materia-, en donde la leyes del empleo juvenil que se constituyan estén integradas a la Ley de Juventud, hasta ahora inexistente, y a la legislación general, en forma complementaria, bajo una visión integral, esto es, a fin de que conformen un cuerpo coherente integrado.

\section{BIBLIOGRAFÍA}

ABDALA, Ernesto, "Innovaciones en la formación profesional desde la óptica de sus repercusiones y efectos en la inserción profesional de los jóvenes”, Formación profesional y empleo, Madrid, Organización de Estados Iberoamericanos, 2013.

Diario Oficial de la Federación, Decreto por el que se otorgan estímulos para promover la incorporación a la seguridad social, 8 de abril de 2014.

, Decreto por el que se otorgan beneficios a quienes tributen en el Régimen de Incorporación Fiscal, 10 de septiembre de 2014.

91 Es cierto que el Programa Nacional de Juventud 2014-2018 comprende una serie de objetivos, estrategias y líneas de acción en materia de empleo juvenil y de la formalización del mismo; sin embargo, se requiere un instrumento exclusivo coordinador en materia de empleo juvenil. 
Esta revista forma parte del acervo de la Biblioteca Jurídica Virtual del Instituto de Investigaciones Jurídicas de la UNAM

INSTITUTO NACIONAL DE ESTAdísTICA, GEOGRAFÍA E INFORMÁTICA (INE-

GI), Encuesta Nacional de Ocupación y Empleo, II trimestre, México, 2013.

—, Encuesta Nacional de Ocupación y Empleo, II trimestre, 2014.

- Estadísticas a propósito del Día Internacional de la Juventud, México, agosto de 2014.

—, México: nuevas estadísticas de informalidad laboral, disponible en: www. inegi.org.mx.

—- Panorámica de la población joven en México desde la perspectiva de su condición de actividad 2013, México, INEGI, 2014.

OIT, Base de datos global de políticas de empleo juvenil, agosto de 2014, disponible en: http:www.ilo.org/employment/tareas/youth-employment/youth-pol/l.

, Conclusiones relativas a la discusión recurrente sobre la protección social (seguridad social), Conferencia Internacional del Trabajo, 100a. reunión, Ginebra, 2011.

- Conclusiones sobre el trabajo decente y la economía informal, Conferencia Internacional del Trabajo, 90a. reunión, Ginebra, 2002.

— El dilema del sector no estructurado, memoria del director general, parte I, Conferencia Internacional del Trabajo, 78 reunión, Ginebra, 1991.

—_, El empleo informal en México: situación actual, políticas y desafíos, Oficina Regional para América Latina y el Caribe, 2014.

—- El trabajo decente y la economia informal, Informe VI, Conferencia Internacional del Trabajo, 90a. reunión, Ginebra, 2002.

—_, Employment, Incomes and Equity: A Strategy for Increasing Productive Employment in Kenya, Ginebra, 1972.

—_, Experiencias recientes de formalización en países de América Latina y el Caribe, Oficina Regional para América Latina y el Caribe, Forlac, 2014.

— Guía de introducción para las organizaciones de empleadores. Combatir los desafíos del empleo de los jóvenes, Centro Internacional de Formación, 2012.

—_, Indicadores clave del mercado de trabajo, 5a. ed., Ginebra, 2007.

—_, La crisis del empleo juvenil: un llamado a la acción, Ginebra, 2012.

—_, La transición de la economía informal a la economía formal, Informe V (1), Conferencia Internacional del Trabajo, 103a. reunión, Ginebra, 2013. 
Esta revista forma parte del acervo de la Biblioteca Jurídica Virtual del Instituto de Investigaciones Jurídicas de la UNAM

—- La transición de la economía informal a la economía formal, Informe V (2), Conferencia Internacional del Trabajo, 103a. reunión, Ginebra, 2013.

- Panorama laboral, Ginebra, 2013.

-, Piso de protección social para una globalización equitativa e inclusiva, Ginebra, 2010.

, Recomendación sobre el desarrollo de los recursos humanos: educación, formación y aprendizaje permanente, 92a. reunión de la Conferencia Internacional del Trabajo, Ginebra, 2004.

—- Resolución relativa al empleo de los jóvenes: vías para acceder a un trabajo decente, Ginebra, 2005.

—-, Resolución sobre el trabajo decente y la economía informal, Ginebra, 2002.

- Seguridad social para justicia social y una globalización equitativa, Informe VI, Conferencia Internacional del Trabajo, 100a. reunión, Ginebra, 2011. 2013. , Trabajo decente y juventud en América Latina. Políticas para la acción, Lima,

, Un buen comienzo: trabajo decente para los jóvenes, Ginebra, 2004.

RODRÍGUEZ, Laura, Políticas públicas para promover el empleo juvenil y el emprendedurismo de los jóvenes en México, 2010.

SÁNCHEZ-CASTAÑEDA, Alfredo, Economía informaly empleo, documento de trabajo, México, UNAM, Instituto de Investigaciones Jurídicas, 2013.

SEDESOL, Programa Nacional de Juventud 2014-2018 PROJUVENTUD, México, IMJUVE.

SHCP, Fomentando la formalidad laboral en todo el territorio nacional, Informe Semanal del Vocero, julio de 2013. TS/ 